\title{
Speckle Tracking for Assessment of Left Ventricular Dyssynchrony
}

\author{
Muhammad Asrar ul Haq1,2, Nima Rudd1,2, Ivan Subiakto', Peter Barlis',2, \\ Nagesh S. Anavekar1,2 \\ ${ }^{1}$ Department of Cardiology, The Northern Hospital, Melbourne, Australia \\ ${ }^{2}$ Department of Medicine, University of Melbourne, Melbourne, Australia \\ Email: muhammad.asrar@unimelb.edu.au
}

Received 28 February 2014; revised 2 April 2014; accepted 10 April 2014

Copyright (C) 2014 by authors and Scientific Research Publishing Inc.

This work is licensed under the Creative Commons Attribution International License (CC BY). http://creativecommons.org/licenses/by/4.0/

(c) (i) Open Access

\begin{abstract}
Cardiac resynchronization therapy (CRT) is an established therapy for selected heart failure (HF) patients to improve symptoms, ventricular function, and survival. Although increasingly used, left ventricular (LV) dyssynchrony assessment by echocardiography has failed to show enough predictive value to assess patient response to CRT and the current guidelines do not recommend its routine use. Furthermore, a variety of echocardiographic techniques used for the purpose, including tissue Doppler imaging (TDI), real time three-dimensional echocardiography, M-mode, and various Doppler parameters, showed limited value and poor agreement between the studies and methods, greatly influenced by interobserver variability. Speckle tracking echocardiography (STE) is a more recent approach that uses strain imaging to assess LV dyssynchrony. This article discusses the speckle tracking for LV dyssynchrony and its current clinical applications.
\end{abstract}

\section{Keywords}

Speckle Tracking, STE, Dyssynchrony, Echocardiography

\section{Introduction}

Cardiac resynchronization therapy (CRT) is an established therapy for selected heart failure (HF) patients to improve symptoms, ventricular function, and survival. Although increasingly used, left ventricular (LV) dyssynchrony assessment by echocardiography has failed to show enough predictive value to assess patient response to CRT and the current guidelines do not recommend its routine use [1]. Furthermore, a variety of echocardiographic techniques used for the purpose, including tissue Doppler imaging (TDI), real time three-dimensional echocardiography, M-mode, and various Doppler parameters, showed limited value and poor agreement be- 
tween the studies and methods, greatly influenced by interobserver and intraobserver variability which has been reported up to 30\% [1]-[3]. Speckle tracking echocardiography (STE) is a more recent approach that uses strain imaging to assess LV dyssynchrony. This article discusses the speckle tracking for LV dyssynchrony and its current clinical applications.

\section{Speckle Tracking, Strain and Strain Rate}

STE works by marking characteristic speckle patterns created by interference of ultrasound beams in the myocardium and then track their individual motion [4]. The technology is able to quantify myocardial motion in different planes, e.g. circumferential and radial, and has been validated non-invasively by magnetic resonance imaging (MRI) tagging and invasively by sonomicrometry in animal models to provide accurate measurements of LV dimensions and strain [5]-[7]. The tracking is based on grey scale B-mode images. This method does not make use of Doppler information, so there is no angle dependency. For accurate speckle tracking, a high frame rate is important as it decrease the speckle change between frames, allowing better tracking.

Strain is a measurement of myocardial deformation. It is defined as dimensionless quantity that represents a percentage change in dimension from resting state to one achieved following application of a force (stress) [8]. It is calculated by tracking the speckles during cardiac cycle for a ratio of the difference in displacement between the two points as compared to their initial distance. Radial, transversal, longitudinal, and circumferential strains can be measured depending on the plane of speckles' motion. Strain rate is simply a rate of this deformation. A positive strain value denotes lengthening whereas a negative value suggests shortening of myocardium.

The advantages of strain or strain rate are that they avoid some of the limitations of wall motion analysis, such as tethering, off-axis false positives and negatives and the difficulties in analysing subtle wall motion. It adds an objective perspective. There are studies showing strain as more sensitive than wall motion for detecting myocardial ischemia [9] [10].

\section{Strain for Cardiac Resynchronization Therapy}

It is estimated that $30 \%$ of patients do not appear to benefit using standard clinical selection criteria [11] [12]. In this context, LV dyssynchrony to predict response to CRT has gained particular interest. Myocardial strain values using both TDI and speckle tracking have been used to assess dyssynchrony. Various studies have assessed TDI derived strain and have established its advantages over other established indexes [1] [13]-[15]. It however is severely limited by the Doppler angle of incidence, a common issue in the enlarged spherical left ventricles in the CRT patients. Consequently, echocardiographic dyssynchrony is currently not considered reliable enough to replace current selection criteria for CRT.

\section{Speckle Tracking for LV Dyssynchrony and Response to CRT}

Four different speckle tracking dyssynchrony approaches have been suggested, including radial and circumferential strain assessed from short-axis views, longitudinal and transverse strain assessed from apical four, three, and two chamber views. Radial and transverse strains have positive curves, reflecting myocardial thickening whereas longitudinal and circumferential strains have negative curves, reflecting myocardial shortening.

Dyssynchrony is typically characterized in LBBB by early septal radial thickening, followed by delayed posterior and lateral wall thickening. The first clinical study to demonstrate that the novel speckle tracking can quantify radial LV dyssynchrony, showed an increase in sensitivity of detecting favourable long term response to CRT [16]. In this study, speckle tracking applied to routine midventricular short-axis images calculated radial strain from multiple circumferential points averaged to 6 standard segments. Baseline speckle-tracking radial dyssynchrony (time difference in peak septal wall-to-posterior wall strain $>$ or $=130 \mathrm{~ms}$ ) predicted an immediate increase in stroke volume the day after CRT with $91 \%$ sensitivity and $75 \%$ specificity. Long-term follow-up (8 +/- 5 months) further showed a significant increase in ejection fraction with $89 \%$ sensitivity and $83 \%$ specificity. Patients in whom left ventricular lead position was concordant with the site of latest mechanical activation by speckle-tracking radial strain had an increase in ejection fraction from baseline to a greater degree than patients with discordant lead position. In another study Lim et al. [17] suggested that strain delay index with longitudinal speckle tracking strain from standard apical views had a strong predictive value for predicting response to CRT in both ischemic and non-ischaemic patients. They demonstrated that the strain delay index $\geq 25 \%$ strongly predicted response to CRT with a $95 \%$ sensitivity and an $83 \%$ specificity and correlated with reverse remodelling in 
both the ischemic $(r=0.68)$ and non-ischaemic $(r=0.68)$ patients $(\mathrm{p}<0.0001$ for both).

Furthermore, STE has the potential to analyse alterations in rotational mechanics caused by dyssynchrony. Sade et al. [18] investigated 54 HF patients and 33 healthy controls to assess the effect of CRT by looking at discoordinate rotation of the apical and basal regions on STE. LV twist was defined as a net difference of LV rotation at isochronal time points between the apical and basal short-axis planes and LV torsion as LV twist divided by LV diastolic longitudinal length. Peak apical and basal rotation, peak LV twist and torsion, apical and basal rotation at aortic valve closure (AVC), and LV twist and torsion at AVC were significantly lower in patients than controls. Apical-basal rotation delay and AVC-to-peak LV twist interval were longer in patients and associated with decreased peak LV twist and LV twist at AVC, respectively. Rotational indexes, particularly LV twist and torsion, were correlated strongly with radial dyssynchrony. LV torsion (cut-off $0.1 \% \mathrm{~cm}$ ) and twist (cut-off $1^{\circ}$ ) at AVC had the highest sensitivity (90\%) and specificity (77\%) to predict CRT responders. The STAR (Speckle Tracking and Resynchronization) study was the first prospective multicentre study to associate speckle tracking strain dyssynchrony with EF response and long-term survival after CRT in 132 patients using radial strain from short-axis views and transverse strain from apical views [19]. Longitudinal and circumferential strain appeared less sensitive. A lack of dyssynchrony before CRT by radial or transverse strain (or both) was significantly associated with death, heart transplantation, or LV assist device implantation. This study also reported the inter- and intra-observer variabilities which were $17+/-14$ and $10+/-6 \%$ for radial dyssynchrony, $18+/-8$ and $11+/-7 \%$ for circumferential dyssynchrony, $17+/-16$ and $11+/-6 \%$ for transverse dyssynchrony, and $19+/-9$ and $13+/-7 \%$ for longitudinal dyssynchrony, respectively.More recently, Delgado et al. [20] showed an association of a lack of dyssynchrony by speckle tracking radial strain with death or heart failure hospitalization in a series of 397 CRT patients with ischemic heart disease. Radial dyssynchrony by speckle tracking strain has been suggested to have the potential to assist with patient selection for CRT with borderline QRS duration [21].

Finally, a recent study derived parameter from the net radial strain delay (RSD) for the 12 basal and mid-left ventricular segments (calculated radial strain delay RSD [RSDc]), based on timing as well as amplitude of segmental strain to enhance the selection CRT [22]. RSDc was calculated as the sum of difference between peak radial strain and radial strain at aortic valve closure before CRT implantation in 240 patients. CRT response was defined as $>15 \%$ reduction in left ventricular end-systolic volume at 6 months. In a derivation group $(\mathrm{n}=102)$, RSDc was higher in responders compared with non-responders and related to the change in left ventricular endsystolic volume. RSDc $>40 \%$ predicted remodelling (sensitivity, 87\%; specificity, $88 \%$ ). In the validation group $(n=108)$, RSDc similarly predicted response (sensitivity, 89\%; specificity, 84\%). Survival at long-term followup was greater in patients with RSDc $>40 \%$. This study demonstrated the value of RSDc, based on both the timing and the amplitude of segmental strain, as a strong predictor for CRT remodelling response and long-term survival.

\section{Further Clinical Applications of STE in LV Dyssynchrony}

\subsection{Right Ventricular Pacing}

Utility of magnetic resonance tagging to assess radial strain and dyssynchrony is limited in the presence of pacemaker. A higher risk of developing HF has been noted in people with permanent right ventricular (RV) pacing. Tops et al. [23] used STE radial strain to determine dyssynchrony in 58 patients with RV pacing. At baseline, similar time-to-peak strain for the 6 segments analysed was observed (mean $371+/-114 \mathrm{~ms}$ ). In contrast, after a mean of $3.8+/-2.0$ years of RV pacing, there was a marked heterogeneity in time-to-peak strain of the 6 segments. In 33 patients (57\%), LV dyssynchrony, represented by a time difference $>$ or $=130$ ms between the time-to-peak strain of the (antero) septal and the posterolateral segments, was present. In these patients, a deterioration of LV systolic function and NYHA functional class was observed. In 11 patients, an "upgrade" of the conventional pacemaker to a biventricular pacemaker resulted in partial reversal of the detrimental effects of RV pacing. This interesting study using STE clearly demonstrated that permanent RV pacing induced heterogeneity in time-to-peak strain, resulting in LV dyssynchrony in $57 \%$ of patients, associated with deterioration of LV systolic function and NYHA functional class and suggested that biventricular pacing may reverse these adverse effects of RV pacing. The study however could not explain why some patients developed significant dyssynchrony while others did not, since all patients had the RV lead positioned at the apex. Exclusion of circumferential and longitudinal strain in this study may have influenced results as well as the use of a six-seg- 
ment algorithm only with possible cause of information loss.

\subsection{Chronic Heart Failure}

Lim et al. [17] have demonstrated a strong predictive value of longitudinal speckle tracking strain for predicting response to CRT in both ischemic and non-ischaemic patients, as mentioned previously. Donal et al. [24] assessed longitudinal and radial dyssynchrony in ischemic and non-ischaemic chronic systolic heart failure in 95 consecutive patients and suggested that the profile of dyssynchrony is influenced by the underlying cause of HF, and that assessment of radial, instead of longitudinal, deformation might be relevant for CRT selection of patients with ischaemic aetiology. The two different aetiologies resulted in different electromechanical correlates. Electrical and mechanical dyssynchrony correlated homogeneously in patients with non-ischaemic heart failure considering radial and longitudinal strain. In ischaemic heart failure radial dyssynchrony was the best mechanical index that correlated with QRS duration.

\subsection{Left Ventricular Hypertrophy}

Nagakura et al. [25] have evaluated dyssynchrony in patients with left ventricular hypertrophy (LVH) comparing abnormalities associated with hypertrophic cardiomyopathy (HCM) and hypertensive heart disease using STE. Three LV short-axis planes were acquired at the basal, middle, and apical levels with high frame rates to obtain radial and circumferential strain. The degree of dyssynchrony noted in patients with hypertensive LVH was within the same range as observed for age-matched controls, while the prevalence and degree of dyssynchrony were significantly higher in patients with HCM, than in those with hypertensive LVH and age-matched controls.

\subsection{Chronic Right Ventricular Pressure and Volume Overload}

Investigation of radial LV-dynamics and dyssynchrony in patients with chronic RV pressure overload revealed a large degree of wall motion dyssynchrony with paradoxical ventricular septal motion, as compared to coordinated radial segmental displacement curves throughout the cardiac cycle in normal subjects [26]. Importantly, radial strain imaging showed uniform radial segmental strain curves, indicating coordinated intrinsic segmental contraction, and relaxation in these patients as well as normal subjects. Impairment of radial synchronicity of segmental wall motion was strongly correlated with LV eccentricity index and myocardial performance index. Similarly, RV volume overload may also be combined with dyssynchrony.

\subsection{Myocardial Infarction}

Severe LV remodelling after myocardial infarction (MI) has been reported impacting significantly on LV synchronicity depending on the infarct size [27] [28]. Mollema et al. [29] investigated dyssynchrony acutely after MI by using radial STE to predict left ventricular remodelling. At 6-month follow-up, end systolic and end diastolic volumes were significantly larger as well as the EF was significantly lower in the patients with LV remodelling which was defined as group A as compared to group B (no LV remodelling at 6 months). The baseline wall motion score index, ratio of mitral E-velocity and early diastolic mitral annulus velocity (E/E'), and dyssynchrony were significantly different between the two groups. Baseline dyssynchrony of $>130 \mathrm{~ms}$ or more had a sensitivity of $82 \%$ and a specificity of $95 \%$ to predict $\mathrm{LV}$ remodelling at 6 -month after acute infarction.

\subsection{Exercise-Induced Torsional Dyssynchrony}

LV systole and diastole are representation of a myocardial rotation (twisting in systole and untwisting in diastole) and longitudinal motion. Heart failure with preserved ejection fraction (HFPEF) is known to involve exerciseinduced wall motion abnormalities and torsion defects [30] [31]. A recent study investigated exercise induced torsional dyssynchrony in 67 patients with HFPEF and 38 controls [32]. Torsional dyssynchrony was quantified as the standard deviation (SD) of the time to peak systolic motion (SDSM) (basal and apical rotation, longitudinal and radial displacement); the time difference between peak twist and peak longitudinal displacement (twistlongitudinal motion delay, TLMD) and the ratio of untwist to longitudinal extension (UT:LE). At rest, HFPEF patients had similar SDSM, TLMD and UT:LE compared with controls. Exercise was associated with significantly more dyssynchrony in the HFPEF patients. The SDSM correlated positively with LV wall thickness, and 
negatively with peak oxygen consumption and changes in stroke volume on exercise, suggesting that HFPEF involves exercise-induced torsional dyssynchrony, which relates to LV hypertrophy as well as exercise capacity.

\section{Three-Dimensional STE}

A newer speckle tracking approach to quantify LV dyssynchrony is 3D speckle tracking echocardiography, which can provide a more comprehensive evaluation of LV mechanics using a 3D model of the entire ventricle for motion analysis [33]-[35]. Tanaka et al. have used a 3-D speckle tracking system to determine radial strain using a 16-segment model from a pyramidal 3-D dataset [36]. 3D cine loops of regional strain were colour coded and divided into 16 segments for time-strain curves, polar maps, and 3D displays. LV dyssynchrony significantly correlated with similar 2-dimensional (2D) strain measures, with 3D having the advantage of more precise mechanical activation mapping to assist with LV lead positioning. 3D speckle tracking strain was also useful for showing differences in the sites of earliest activation in right ventricle-paced patients compared with those with LBBB, but similar sites of latest activation and similar response to CRT [37].

In another recent study real-time 3D speckle tracking echocardiography (3DSTE) was used as a novel method to assess dyssynchrony [38]. 3D radial, longitudinal, as well as novel 3D area strain (AS) were performed on 60 unselected patients who were referred to optimise and to control of a CRT device. AS was based on interface (endocardium or epicardium) tracking, reflecting the deformation of a box during contraction and relaxation. Given the area definition of product of length and width, AS can be considered a combination of the total vector resultant based on radial, circumferential, and longitudinal vectors. In the comparison of utility of the mentioned various 3D strain measurements, the authors suggested that 3D AS appeared to be close to the ideal parameter and allowed a rapid and global assessment of LV dyssynchrony. Further studies using this "multi-directional parameter” of function and dyssynchrony are needed.

\section{Conclusion}

Speckle tracking is a powerful technology for an objective assessment of dyssynchrony. Multiple studies have demonstrated the potential of this novel technology to aid in patient selection for CRT, post CRT follow-up, and various other myocardial pathologies. Limitations include requirement of high quality 2D images since poor speckle tracking can lead to false-positive results. A possible loss of speckles due to moving out of the 2D imaging plane may influence the measurements. Novel 3D speckle tracking may overcome this problem. 3D speckle tracking adds new dimensions and is at the forefront of a new area of clinical research.

\section{References}

[1] Chung, E.S., et al. (2008) Results of the Predictors of Response to CRT (PROSPECT) Trial. Circulation, 117, 2608-2616. http://dx.doi.org/10.1161/CIRCULATIONAHA.107.743120

[2] Burri, H., et al. (2008) Poor Agreement of Echographic Measures of Ventricular Dyssynchrony. European Journal of Echocardiography, 9, 235-240.

[3] Burgess, M.I., et al. (2007) Measurement of Left Ventricular Dyssynchrony in Patients with Ischaemic Cardiomyopathy: A Comparison of Real-Time Three-Dimensional and Tissue Doppler Echocardiography. Heart, 93, 1191-1196. http://dx.doi.org/10.1136/hrt.2006.101626

[4] Bohs, L.N. and Trahey, G.E. (1991) A Novel Method for Angle Independent Ultrasonic Imaging of Blood Flow and Tissue Motion. IEEE Transactions on Biomedical Engineering, 38, 280-286. http://dx.doi.org/10.1109/10.133210

[5] Amundsen, B.H., et al. (2006) Noninvasive Myocardial Strain Measurement by Speckle Tracking Echocardiography: Validation against Sonomicrometry and Tagged Magnetic Resonance Imaging. Journal of the American College of Cardiology, 47, 789-793. http://dx.doi.org/10.1016/j.jacc.2005.10.040

[6] Reisner, S.A., et al. (2004) Global Longitudinal Strain: A Novel Index of Left Ventricular Systolic Function. Journal of the American Society of Echocardiography, 17, 630-633. http://dx.doi.org/10.1016/j.echo.2004.02.011

[7] Leitman, M., et al. (2004) Two-Dimensional Strain-A Novel Software for Real-Time Quantitative Echocardiographic Assessment of Myocardial Function. Journal of the American Society of Echocardiography, 17, 1021-1029. http://dx.doi.org/10.1016/j.echo.2004.06.019

[8] Mirsky, I. and Parmley, W.W. (1973) Assessment of Passive Elastic Stiffness for Isolated Heart Muscle and the Intact Heart. Circulation Research, 33, 233-243. http://dx.doi.org/10.1161/01.RES.33.2.233

[9] Winter, R., et al. (2007) Speckle Tracking Echocardiography Is a Sensitive Tool for the Detection of Myocardial 
Ischemia: A Pilot Study from the Catheterization Laboratory during Percutaneous Coronary Intervention. Journal of the American Society of Echocardiography, 20, 974-981. http://dx.doi.org/10.1016/j.echo.2007.01.029

[10] Ng, A.C.T., et al. (2009) Incremental Value of 2-Dimensional Speckle Tracking Strain Imaging to Wall Motion Analysis for Detection of Coronary Artery Disease in Patients Undergoing Dobutamine Stress Echocardiography. American Heart Journal, 158, 836-844. http://dx.doi.org/10.1016/j.ahj.2009.09.010

[11] Abraham, W.T., et al. (2002) Cardiac Resynchronization in Chronic Heart Failure. The New England Journal of Medicine, 346, 1845-1853. http://dx.doi.org/10.1056/NEJMoa013168

[12] Cleland, J.G., et al. (2005) The Effect of Cardiac Resynchronization on Morbidity and Mortality in Heart Failure. The New England Journal of Medicine, 352, 1539-1549. http://dx.doi.org/10.1056/NEJMoa050496

[13] Gorcsan, J., et al. (2010) Relationship of Echocardiographic Dyssynchrony to Long-Term Survival after Cardiac Resynchronization Therapy. Circulation, 122, 1910-1918. http://dx.doi.org/10.1161/CIRCULATIONAHA.110.954768

[14] Miyazaki, C., et al. (2008) Comparison of Echocardiographic Dyssynchrony Assessment by Tissue Velocity and Strain Imaging in Subjects with or without Systolic Dysfunction and with or without Left Bundle-Branch Block. Circulation, 117, 2617-2625. http://dx.doi.org/10.1161/CIRCULATIONAHA.107.733675

[15] Yu, C.M., et al. (2007) Usefulness of Tissue Doppler Velocity and Strain Dyssynchrony for Predicting Left Ventricular Reverse Remodeling Response after Cardiac Resynchronization Therapy. American Journal of Cardiology, 100, 1263-1270. http://dx.doi.org/10.1016/j.amjcard.2007.05.060

[16] Suffoletto, M.S., Dohi, K., Cannesson, M., Saba, S. and Gorcsan III, J. (2006) Novel Speckle-Tracking Radial Strain from Routine Black-and-White Echocardiographic Images to Quantify Dyssynchrony and Predict Response to Cardiac Resynchronization Therapy. Circulation, 113, 960-968. http://dx.doi.org/10.1161/CIRCULATIONAHA.105.571455

[17] Lim, P., Buakhamsri, A., Popovic, Z.B., Greenberg, N.L., Patel, D., Thomas, J.D. and Grimm, R.A. (2008) Longitudinal Strain Delay Index by Speckle Tracking Imaging: A New Marker of Response to Cardiac Resynchronization Therapy. Circulation, 118, 1130-1137. http://dx.doi.org/10.1161/CIRCULATIONAHA.107.750190

[18] Sade, L.E., Demir, Ö., Atar, I., Müderrisoglu, H. and Özin, B. (2008) Effect of Mechanical Dyssynchrony and Cardiac Resynchronization Therapy on Left Ventricular Rotational Mechanics. American Journal of Cardiology, 101, 11631169. http://dx.doi.org/10.1016/j.amjcard.2007.11.069

[19] Tanaka, H., Nesser, H.J., Buck, T., Oyenuga, O., Alexander Jánosi, R., Winter, S., Saba, S. and Gorcsan III, J. (2010) Dyssynchrony by Speckle-Tracking Echocardiography and Response to Cardiac Resynchronization Therapy: Results of the Speckle Tracking and Resynchronization (STAR) Study. European Heart Journal, 31, 1690-1700. http://dx.doi.org/10.1093/eurheartj/ehq213

[20] Delgado, V., van Bommel, R.J., Bertini, M., et al. (2011) Relative Merits of Left Ventricular Dyssynchrony, Left Ventricular Lead Position, and Myocardial Scar to Predict Long-Term Survival of Ischemic Heart Failure Patients Undergoing Cardiac Resynchronization Therapy. Circulation, 123, 70-78. http://dx.doi.org/10.1161/CIRCULATIONAHA.110.945345

[21] Oyenuga, O., Hara, H., Tanaka, H., Kim, H.N., Adelstein, E.C., Saba, S. and Gorcsan III, J. (2010) Usefulness of Echocardiographic Dyssynchrony in Patients with Borderline QRS Duration to Assist with Selection for Cardiac Resynchronization Therapy. JACC: Cardiovascular Imaging, 3, 132-140. http://dx.doi.org/10.1016/j.jcmg.2009.09.020

[22] Kydd, A.C., Khan, F.Z., O’Halloran, D., Pugh, P.J., Virdee, M.S. and Dutka, D.P. (2013) Radial Strain Delay Based on Segmental Timing and Strain Amplitude Predicts Left Ventricular Reverse Remodeling and Survival after Cardiac Resynchronization Therapy. Circulation: Cardiovascular Imaging, 6, 177-184. http://dx.doi.org/10.1161/CIRCIMAGING.112.000191

[23] Tops, L.F., Suffoletto, M.S., Bleeker, G.B., Boersma, E., van der Wall, E.E., Gorcsan III, J., Schalij, M.J. and Bax, J.J. (2007) Speckle-Tracking Radial Strain Reveals Left Ventricular Dyssynchrony in Patients with Permanent Right Ventricular Pacing. Journal of the American College of Cardiology, 50, 1180-1188. http://dx.doi.org/10.1016/j.jacc.2007.06.011

[24] Donal, E., Tournoux, F., Leclercq, C., De Place, C., Solnon, A., Derumeaux, G., Mabo, P., Cohen-Solal, A. and Daubert, J.C. (2008) Assessment of Longitudinal and Radial Ventricular Dyssynchrony in Ischemic and Nonischemic Chronic Systolic Heart Failure: A Two-Dimensional Echocardiographic Speckle-Tracking Strain Study. Journal of the American Society of Echocardiography, 21, 58-65. http://dx.doi.org/10.1016/j.echo.2007.05.031

[25] Nagakura, T., Takeuchi, M., Yoshitani, H., Nakai, H., Nishikage, T., Kokumai, M., Otani, S., Yoshiyama, M. and Yoshikawa, J. (2007) Hypertrophic Cardiomyopathy Is Associated with More Severe Left Ventricular Dyssynchrony than Is Hypertensive Left Ventricular Hypertrophy. Echocardiography, 24, 677-684. http://dx.doi.org/10.1111/j.1540-8175.2007.00458.x

[26] Dohi, K., Onishi, K., Gorcsan III, J., López-Candales, A., Takamura, T., Ota, S., Yamada, N. and Ito, M. (2008) Role of Radial Strain and Displacement Imaging to Quantify Wall Motion Dyssynchrony in Patients with Left Ventricular 
Mechanical Dyssynchrony and Chronic Right Ventricular Pressure Overload. American Journal of Cardiology, 101, 1206-1212. http://dx.doi.org/10.1016/j.amjcard.2007.11.077

[27] Giannuzzi, P., Temporelli, P.L., Bosimini, E., Gentile, F., Lucci, D., Maggioni, A.P., Tavazzi, L., Badano, L., Stoian, I., Piazza, R., Heyman, I., Levantesi, G., Cervesato, E., Geraci, E. and Nicolosi, G.L. (2001) Heterogeneity of Left Ventricular Remodeling after Acute Myocardial Infarction: Results of the Gruppo Italiano per lo Studio della Sopravvivenza nell’Infarto Miocardico-3 Echo Substudy. American Heart Journal, 141, 131-138. http://dx.doi.org/10.1067/mhj.2001.111260

[28] Zhang, Y., Chan, A.K.Y., Yu, C.M., Lam, W.W.M., Yip, G.W.K., Fung, W.H., So, N.M.C., Wang, M. and Sanderson, J.E. (2005) Left Ventricular Systolic Asynchrony after Acute Myocardial Infarction in Patients with Narrow QRS Complexes. American Heart Journal, 149, 497-503. http://dx.doi.org/10.1016/j.ahj.2004.05.054

[29] Mollema, S.A., Liem, S.S., Suffoletto, M.S., Bleeker, G.B., van der Hoeven, B.L., van de Veire, N.R., Boersma, E., Holman, E.R., van der Wall, E.E., Schalij, M.J., Gorcsan III, J. and Bax, J.J. (2007) Left Ventricular Dyssynchrony Acutely after Myocardial Infarction Predicts Left Ventricular Remodeling. Journal of the American College of Cardiology, 50, 1532-1540. http://dx.doi.org/10.1016/j.jacc.2007.07.025

[30] Lin, T., Asrar ul Haq, M. and Wong, C. (2009) Abstract 215: Two-Dimensional Speckle Tracking Echocardiography to Assess Changes in Left Ventricular Torsional Dynamics During Exercise Is a Better Predictor of Exercise Capacity and Myocardial Dysfunction Compared with E/E’ Estimation. Circulation, 120, S303.

[31] Asrar ul Haq, M., Mutha, V., Lin, T., Profitis, K., Tuer, Z., Lim, K., Hare, D.L. and Wong, C. (2014) Left Ventricular Torsional Dynamics Post Exercise for LV Diastolic Function Assessment. Cardiovascular Ultrasound, $12,8$.

[32] Tan, Y.T., Wenzelburger, F.W.G., Sanderson, J.E. and Leyva, F. (2013) Exercise-Induced Torsional Dyssynchrony Relates to Impaired Functional Capacity in Patients with Heart Failure and Normal Ejection Fraction. Heart, 99, 259266. http://dx.doi.org/10.1136/heartjnl-2012-302489

[33] Chen, X., Xie, H., Erkamp, R., Kim, K., Jia, C., Rubin, J.M. and O’Donnell, M. (2005) 3-D Correlation-Based Speckle Tracking. Ultrasonic Imaging, 27, 21-36. http://dx.doi.org/10.1177/016173460502700102

[34] Meunier, J. (1998) Tissue Motion Assessment from 3D Echographic Speckle Tracking. Physics in Medicine and Biology, 43, 1241-1254. http://dx.doi.org/10.1088/0031-9155/43/5/014

[35] Myronenko, A., Song, X. and Sahn, D.J. (2007) LV Motion Tracking from 3D Echocardiography Using Textural and Structural Information. Medical Image Computing and Computer-Assisted Intervention, 10, 428-435.

[36] Tanaka, H., Hara, H., Saba, S. and Gorcsan, J. (2010) Usefulness of Three-Dimensional Speckle Tracking Strain to Quantify Dyssynchrony and the Site of Latest Mechanical Activation. American Journal of Cardiology, 105, $235-242$. http://dx.doi.org/10.1016/j.amjcard.2009.09.010

[37] Tanaka, H., Hara, H., Adelstein, E.C., Schwartzman, D., Saba, S. and Gorcsan III, J. (2010) Comparative Mechanical Activation Mapping of RV Pacing to LBBB by 2D and 3D Speckle Tracking and Association with Response to Resynchronization Therapy. JACC: Cardiovascular Imaging, 3, 461-471. http://dx.doi.org/10.1016/j.jcmg.2009.12.014

[38] Thebault, C., Donal, E., Bernard, A., Moreau, O., Schnell, F., Mabo, P. and Leclercq, C. (2011) Real-Time Three-Dimensional Speckle Tracking Echocardiography: A Novel Technique to Quantify Global Left Ventricular Mechanical Dyssynchrony. European Journal of Echocardiography, 12, 26-32. http://dx.doi.org/10.1093/ejechocard/jeq095 